\title{
ON THE REGULARITY OF SOLUTIONS OF A HOMOGENEOUS DIRICHLET PROBLEM FOR A NON-HYPOELLIPTIC LINEAR PARTIAL DIFFERENTIAL OPERATOR
}

\author{
K. Doppel and R. Hochmuth
}

\section{Introduction}

1.1. In the study of the regularity of generalized solutions of various problems for partial differential equations the notion of the hypoellipticity of linear partial differential operators has a central role. Originally the hypoelliptic operators were introduced by L. Hörmander in 1955, and for the results of the development one can today consult his monograph [13], especially chapters 11 and 13.

A linear differential operator $P(\cdot, D)$ defined in some open set $\Omega$ in $\mathbf{R}^{n}$ through

$$
P(x, D) u=\sum_{|\alpha| \leq m} a_{\alpha}(x) D^{\alpha} u
$$

with given coefficients $a_{\alpha} \in C^{\infty}(\Omega)$ is called hypoelliptic if the relation

$$
\text { sing supp } u=\operatorname{sing} \operatorname{supp} P(\cdot, D) u
$$

is valid for all distributions $u \in \mathcal{D}^{\prime}(\Omega)$.

Especially in the case of a differential operator

$$
P(D) u=\sum_{|\alpha| \leq m} c_{\alpha} D^{\alpha}
$$

with constant coefficients $c_{\alpha} \in \mathbf{C}$ there are several equivalent characterizations of the hypoellipticity, among them the following: The differential operator (1.3) is hypoelliptic in $\mathbf{R}^{n}$ (and in every open subset $\Omega$ of $\mathbf{R}^{n}$ ) if and only if for all $\alpha \in \mathbf{N}_{0}^{n}$ and for $|\xi| \rightarrow \infty\left(\xi \in \mathbf{R}^{n}\right)$

$$
P^{(\alpha)}(i \xi) / P(i \xi) \rightarrow 0
$$

(cf. Hörmander [13], Theorem 11.1.1, p. 61). 
From the property (1.2) it becomes obvious that for generalized solutions $u$ (e.g. for $\left.u \in \mathcal{D}^{\prime}(\Omega)\right)$ of the differential equation

$$
P(x, D) u=f
$$

with a hypoelliptic differential operator $P(\cdot, D)$ one gets from local regularity properties of the given right-hand side function $f$ (say, from $f \in C^{\infty}\left(\Omega_{1}\right)$ with $\left.\Omega_{1} \subset \subset \Omega\right)$ the local regularity of $u\left(u \in C^{\infty}\left(\Omega_{1}\right)\right)$.

Thus, we have every reason to expect to get for generalized solutions of different boundary value problems for hypoelliptic differential operators (and also for hypoelliptic pseudo-differential operators) regularity results similar to or somewhat weaker than previously known for elliptic operators. Many authors have published works on the research in this direction.

At the same time the question arises whether it is possible to prove, at least in some special cases, regularity results for generalized solutions of problems for differential operators which are not hypoelliptic. From the short remarks above it should be clear that such results could still be possible to reach if one assumes global regularity properties of the given data. As far as we know, only a few results in this direction have been published so far.

1.2. In a series of papers, I.S. Louhivaara and C.G. Simader studied $t$ coercive linear partial differential operators of order $m(m \in \mathbf{N}, t \in \mathbf{N}$ or $t \in \mathbf{R}$, $0<t \leq m)$. There are also non-hypoelliptic differential operators in these classes.

In 1972 they proved the following regularity result [15]: Let $P(D)$ be a $2 t$ coercive linear differential operator of order $m$ with constant coefficients $(m, t \in$ $\mathbf{N}, 2 t \leq m)$. If $u \in L^{2}\left(\mathbf{R}^{n}\right)$ is a weak $L^{2}$-solution in $\mathbf{R}^{n}$ of the differential equation $P(D) u=f$ with $f \in H^{k}\left(\mathbf{R}^{n}\right)(k \in \mathbf{N})$, i.e. if $u$ satisfies with the formal adjoint differential operator $P^{\prime}(D)$ the relation

$$
\left(u, P^{\prime}(D) \varphi\right)_{L^{2}\left(\mathbf{R}^{n}\right)}=(f, \varphi)_{L^{2}\left(\mathbf{R}^{n}\right)}
$$

for all $\varphi \in C_{0}^{\infty}\left(\mathbf{R}^{n}\right)$, then $u \in H^{2 t+k}\left(\mathbf{R}^{n}\right)$.

Furthermore, in 1978 the same authors [16] proved a corresponding result for periodic solutions of a $t$-coercive differential operator $(t \in \mathbf{R}, t>0)$ even in the case of non-constant coefficients periodic in all variables.

We also mention the works by S.J. Greenfield and N.R. Wallach [9], D. Fujiwara and H. Omori [8], and M. Yoshino [23]. They all investigate on the $n$ dimensional torus some classes of differential operators which also include operators not hypoelliptic in the above sense but yet "globally hypoelliptic".

1.3. In this paper we shall study the regularity of the solutions of a homogeneous Dirichlet problem for a bounded domain $\Omega=\Omega_{1} \times \Omega_{2}$ in $\mathbf{R}^{n}$ with $\Omega_{\mu} \subset \mathbf{R}^{n_{\mu}}$ 
$\left(n_{\mu} \geq 2, \mu \in\{1,2\}\right)$ and $n=n_{1}+n_{2}$ and for a linear partial differential operator which is a product of two uniformly elliptic differential operators with smooth coefficients defined in $\Omega_{1}$ and $\Omega_{2}$. The existence and uniqueness of the generalized solutions of this problem in the case of a product of Laplace operators was shown in the paper [4] by one of the present authors together with N. Jacob.

The idea of such Dirichlet problems comes from the research [6] of E.B. Dynkin on the simplest random fields to several independent Markov processes observed at different times. He even gave a proof for the existence of a solution of the Dirichlet problem by methods developed in stochastic analysis.

In this connection we also refer to the papers of H.-J. Herrler [10-11], K. Doppel and N. Jacob [5], N. Jacob [14], and B. Schomburg [21]. We would also like to remark that D. Mangeron [17] already in 1933 studied, by totally classical methods, a Dirichlet problem where the differential operator could be interpreted as a special product operator in the sense of [6], [4] and of this paper.

As an example of differential operators to be studied in this note, let us take the simplest case $n_{1}=n_{2}=2$ and

$$
P(D)=\left(\frac{\partial^{2}}{\partial x_{1}^{2}}+\frac{\partial^{2}}{\partial x_{2}^{2}}\right) \circ\left(\frac{\partial^{2}}{\partial x_{3}^{2}}+\frac{\partial^{2}}{\partial x_{4}^{2}}\right) .
$$

The symbol associated with $P(D)$ is given by $P(i \xi)=\xi_{1}^{2} \xi_{3}^{2}+\xi_{2}^{2} \xi_{3}^{2}+\xi_{1}^{2} \xi_{4}^{2}+\xi_{2}^{2} \xi_{4}^{2}$. Since for $\xi_{1}=1 / k, \xi_{3}=k, k \in \mathbf{N}$, and $\xi_{2}=\xi_{4}=0$ we have $D^{(2,0,0,0)} P(i \xi) / P(i \xi)$ $=2 k^{2}$, it follows by the above-mentioned result (1.4) of L. Hörmander that $P(D)$ is not hypoelliptic.

Thus the class of differential operators $P\left(\cdot, D_{x}\right)$ we are studying here includes non-hypoelliptic differential operators. However, we are able to show that in suitable bounded product domains $\Omega$ the homogeneous Dirichlet problem behaves hypoelliptically, i.e. it is possible to conclude the smoothness of the solution in $\bar{\Omega}$ from the smoothness of the right-hand side of the differential equation in $\bar{\Omega}$ and from the smoothness of the coefficients of $P\left(\cdot, D_{x}\right)$.

Thus we shall show that there are "globally hypoelliptic" homogeneous Dirichlet problems for non-hypoelliptic partial differential operators.

\section{Definitions and the statement of the problem}

2.1. By $\mathbf{N}_{0}^{m}$ we denote the set of all ordered systems of $m$ nonnegative integers (multi-indices). For $\alpha=\left(\alpha_{1}, \ldots, \alpha_{m}\right) \in \mathbf{N}_{0}^{m}$ we define its length as usual by $|\alpha|:=\alpha_{1}+\cdots+\alpha_{m}$. Especially, we define for each $i \in\{1, \ldots, m\}$ the multi-index $\varepsilon_{i} \in \mathbf{N}_{0}^{m}$ by $\left|\varepsilon_{i}\right|=1$ and the entry 1 in the $i$-th position. For each multi-index $\alpha=\left(\alpha_{1}, \ldots, \alpha_{m}\right) \in \mathbf{N}_{0}^{m}$ we define the partial differential operator $D^{\alpha}$ by $D^{\alpha}:=\partial^{|\alpha|} / \partial x_{1}{ }^{\alpha_{1}} \ldots \partial x_{m}{ }^{\alpha_{m}}$, and for $\varepsilon_{i}$ with $i \in\{1, \ldots, m\}$ we write $D_{i}:=D^{\varepsilon_{i}}$.

For the euclidean norm of $x=\left(x_{1}, \ldots, x_{m}\right) \in \mathbf{R}^{m}$ we use the notation $|x|:=$ $\left(x_{1}^{2}+\cdots+x_{m}^{2}\right)^{1 / 2}$. 
Let $T$ be an arbitrary open set in $\mathbf{R}^{m}, m \in \mathbf{N}$. By $\bar{T}$ we denote the closure of $T$ with respect to the euclidean norm in $\mathbf{R}^{m}$ and by $\partial T:=\bar{T} \backslash T$ we denote the boundary of $T$. For $k \in \mathbf{N}_{0}$ the set $C^{k}(T)$ consists of the functions $u: T \rightarrow \mathbf{R}$, which possess in $T$ continuous derivatives up to the order $k$, i.e., for all $\alpha \in \mathbf{N}_{0}^{m}$ with $|\alpha| \leq k$ there exists a partial derivative $D^{\alpha} u$ and this is continuous in $T$. By $C^{k}(\bar{T})$ we mean the space of functions $u \in C^{k}(T)$ that possess with their derivatives, up to the order $k$, continuous extensions to the closure $\bar{T}$. Instead of $C^{0}(\bar{T})$ we write $C(\bar{T})$.

A function $u: T \rightarrow \mathbf{R}$ is called Hölder continuous to the exponent $\lambda \in(0,1]$ if there exists a constant $C \in \mathbf{R}^{+}$with

$$
\frac{|u(x)-u(y)|}{|x-y|^{\lambda}} \leq C
$$

for all $x, y \in T$ with $x \neq y$. Consequently, $C^{0 ; \lambda}(T)$ denotes the subspace of $C(\bar{T})$ consisting of all functions which are Hölder continuous to the exponent $\lambda$.

We further set

$$
C^{\infty}(\bar{T}):=\bigcap_{t \in N_{0}} C^{t}(\bar{T})
$$

and define $C_{0}^{\infty}(T)$ as the space of all functions $u \in C^{\infty}(\bar{T})$ with compact support in $T$.

Now we define for $k \in \mathbf{N}_{0}$

$$
C_{*}^{k}(T):=\left\{u \in C^{k}(T) \mid D^{\alpha} u \in L^{2}(T) \text { for all } \alpha \in \mathbf{N}_{0}^{m} \text { with }|\alpha| \leq k\right\} .
$$

With the usual scalar product in $L^{2}(T)$,

$$
(u, v)_{0 ; T}=\int_{T} u(x) v(x) d x
$$

we define in $C_{*}^{k}(T)$ by

$$
(u, v)_{k ; T}:=\left(\sum_{|\alpha| \leq k}\left(D^{\alpha} u, D^{\alpha} v\right)_{0 ; T}\right)^{1 / 2}, \quad u, v \in C_{*}^{k}(T),
$$

a scalar product with the corresponding norm $\|\cdot\|_{k ; T}$. The completion of $C_{*}^{k}(T)$ with respect to $(\cdot, \cdot)_{k ; T}$ will be denoted by $H^{k}(T)$.

Define the set of all real-valued measurable locally summable functions $u$ on $T$ by $L_{\text {loc }}^{1}(T)$. As usual, for $\alpha \in \mathrm{N}_{0}^{m}$ we call a function $v_{\alpha} \in L_{\text {loc }}^{1}(T)$ the weak derivative of $u \in L_{\text {loc }}^{1}(T)$ if

$$
\left(u, D^{\alpha} \varphi\right)_{0 ; T}=(-1)^{|\alpha|}\left(v_{\alpha}, \varphi\right)_{0 ; T}
$$

is valid for all $\varphi \in C_{0}^{\infty}(T)$. In this case we write $\partial^{\alpha} u:=v_{\alpha}$. The space $W^{k}(T)$ is then defined by

$$
W^{k}(T):=\left\{u \in L^{2}(T) \mid \partial^{\alpha} u \in L^{2}(T) \text { for all } \alpha \in \mathbf{N}_{0}^{m} \text { with }|\alpha| \leq k\right\},
$$

and it is well known that the spaces $H^{k}(T)$ and $W^{k}(T)$ coincide (cf. Adams [1], Theorem 3.16, p. 52). 
2.2. For arbitrary open sets $\Omega_{1} \subset \mathbf{R}^{n_{1}}$ and $\Omega_{2} \subset \mathbf{R}^{n_{2}}\left(n_{1}, n_{2} \geq 2\right)$ we define $\Omega=\Omega_{1} \times \Omega_{2} \in \mathbf{R}^{n}\left(n=n_{1}+n_{2}\right)$. By $\mathcal{N}^{\mu}(\mu \in\{1,2\})$ we denote the sets of all such multi-indices $\alpha^{\mu}=\left(\alpha_{1}^{\mu}, \ldots, \alpha_{n}^{\mu}\right) \in \mathbf{N}_{0}^{n}$ for which $\alpha_{j}^{1}=0$ for all $j \in\left\{n_{1}+1, \ldots, n\right\}$ and $\alpha_{k}^{2}=0$ for all $k \in\left\{1, \ldots, n_{1}\right\}$, respectively.

For $s, t \in \mathbf{N}_{0}$ let $C^{s, t}(\Omega)$ be the linear space of all functions $u: \Omega \rightarrow \mathbf{R}$ whose partial derivatives $D^{\beta} u$ for $\beta=\alpha^{1}+\alpha^{2}$ with $\alpha^{\mu} \in \mathcal{N}^{\mu},\left|\alpha^{1}\right| \leq s$ and $\left|\alpha^{2}\right| \leq t$ exist in $\Omega$ and are continuous. The linear space of functions $u \in C^{s, t}(\Omega)$ for which the derivatives $D^{\beta} u$ with $\beta=\alpha^{1}+\alpha^{2}, \alpha^{\mu} \in \mathcal{N}^{\mu},\left|\alpha^{1}\right| \leq s,\left|\alpha^{2}\right| \leq t$ belong to $C^{0 ; \lambda}(\Omega)(\lambda \in(0,1])$ will be denoted by $C^{s, t ; \lambda}(\Omega)$.

We further define the (anisotropic) Sobolev space $H^{s, t}(\Omega)\left(s, t \in \mathbf{N}_{0}\right)$ as the completion of $C_{*}^{s+t}(\Omega)$ with respect to the norm

$$
\|u\|_{s, t ; \Omega}:=\left(\sum_{\substack{\alpha=\alpha^{1}+\alpha^{2} \\\left|\alpha^{1}\right| \leq s,\left|\alpha^{2}\right| \leq t}}\left\|D^{\alpha} u\right\|_{0 ; \Omega}^{2}\right)^{1 / 2}
$$

and $H_{0}^{1,1}(\Omega)$ as the smallest closed subspace of $H^{1,1}(\Omega)$ including $C_{0}^{\infty}(\Omega)$. Analogously to subsection 2.1 we define $W^{s, t}(\Omega)$ by

$W^{s, t}(\Omega):=$

$\left\{u \in L^{2}(\Omega) \mid \partial^{\alpha} u \in L^{2}(\Omega)\right.$ for all $\alpha=\alpha^{1}+\alpha^{2}$ with $\left.\alpha^{\mu} \in \mathcal{N}^{\mu},\left|\alpha^{1}\right| \leq s,\left|\alpha^{2}\right| \leq t\right\}$.

Then we have

Theorem 2.1. The spaces $H^{s, t}(\Omega)$ and $W^{s, t}(\Omega)$ are equal.

For the proof see Adams [1], Theorem 3.16, p. 52, and Hochmuth [12], Satz 4.3 , p. 59. We remark that, for star-shaped domains, J. Rákosník has proved this result for more general anisotropic Sobolev spaces (cf. [19], Theorem 2, p. 60).

2.3. Let now $\Omega_{1}$ and $\Omega_{2}$ be bounded domains in $\mathbf{R}^{n_{1}}$ and $\mathbf{R}^{n_{2}}\left(n_{1}, n_{2} \geq 2\right)$ with boundaries $\partial \Omega_{\mu}(\mu \in\{1,2\})$ of class $C^{\infty}$ (cf. e.g. Wloka [22], p. 46). Thus the domains $\Omega_{\mu}$ satisfy the uniform cone condition and the product domain $\Omega:=\Omega_{1} \times \Omega_{2} \subset \mathbf{R}^{n}\left(n=n_{1}+n_{2}\right)$ has the same property (cf. Hochmuth [12], Satz 3.1, p. 21).

On each of the domains $\Omega_{\mu}$ we consider a uniformly elliptic differential operator $P_{\mu}\left(\cdot, D_{x_{\mu}}\right)$,

$$
P_{\mu}\left(\cdot, D_{x_{\mu}}\right):=\sum_{i, j=1}^{n_{\mu}}-D_{j}\left(a_{i j}^{(\mu)}(\cdot) D_{i}\right)+\sum_{i=1}^{n_{\mu}} b_{i}^{(\mu)}(\cdot) D_{i}+c^{(\mu)}(\cdot),
$$


where $a_{i j}^{(\mu)}, b_{i}^{(\mu)}, c^{(\mu)} \in C^{\infty}\left(\bar{\Omega}_{\mu}\right)$ are given real-valued functions with $a_{i j}^{(\mu)} \equiv a_{j i}^{(\mu)}$. Note that then there are constants $\varrho_{\mu} \in \mathbf{R}^{+}$with

$$
\sum_{i, j=1}^{n_{\mu}} a_{i j}^{(\mu)}\left(x_{\mu}\right) \xi_{i} \xi_{j} \geq \varrho_{\mu} \sum_{i=1}^{n_{\mu}} \xi_{i}^{2} \quad \text { for all } x_{\mu} \in \Omega_{\mu} \text { and }\left(\xi_{1}, \ldots, \xi_{n_{\mu}}\right) \in \mathbf{R}^{n_{\mu}}
$$
by

On the product domain $\Omega=\Omega_{1} \times \Omega_{2}$ we define the product operator $P\left(\cdot, D_{x}\right)$

$$
P\left(x, D_{x}\right):=P_{1}\left(x_{1}, D_{x_{1}}\right) P_{2}\left(x_{2}, D_{x_{2}}\right) \quad \text { for } x=\left(x_{1}, x_{2}\right) \in \Omega_{1} \times \Omega_{2} .
$$

For the product operator $P\left(\cdot, D_{x}\right)$ we consider the following Dirichlet problem in the product domain $\Omega$.

Problem $(\Omega)$. For $f \in C(\Omega)$ find a function $u \in C^{2,2}(\Omega) \cap C^{1}(\bar{\Omega})$ such that

$$
\begin{array}{rlrl}
P\left(x, D_{x}\right) u(x) & =f(x) \quad \text { for } x \in \Omega, \\
D^{\alpha^{1}} u(x) & =0 & \text { for } x \in \Omega_{1} \times \partial \Omega_{2}, \\
D^{\alpha^{2}} u(x) & =0 & \text { for } x \in \partial \Omega_{1} \times \Omega_{2}
\end{array}
$$

is valid for all $\alpha^{\mu} \in \mathcal{N}^{\mu}$ with $\left|\alpha^{1}\right|,\left|\alpha^{2}\right| \leq 1$.

We remark that for each solution of Problem $(\Omega)$ the assumption $u \in C^{1}(\bar{\Omega})$ and the boundary conditions (2.7), (2.8) give $D^{\alpha^{1}} u(x)=D^{\alpha^{2}} u(x)=0$ for all $\alpha^{\mu} \in \mathcal{N}^{\mu}$ with $\left|\alpha^{1}\right|,\left|\alpha^{2}\right| \leq 1$ at the boundary points $x \in \partial \Omega_{1} \times \partial \Omega_{2}$.

2.4. To give a weak formulation of Problem $(\Omega)$ we define

$$
\Gamma:=\left\{\sigma_{1} \in \mathbf{N}_{0}^{n_{1}}|| \sigma_{1} \mid \leq 1\right\} \times\left\{\sigma_{2} \in \mathbf{N}_{0}^{n_{2}}|| \sigma_{2} \mid \leq 1\right\} .
$$

A simple calculation shows that there are functions $a_{\sigma \tau} \in C^{\infty}(\bar{\Omega})(\sigma, \tau \in \Gamma)$ with which we can write the operator $P\left(\cdot, D_{x}\right)$ in the form

$$
P\left(\cdot, D_{x}\right)=\sum_{\sigma, \tau \in \Gamma}(-1)^{|\sigma|} D^{\sigma}\left(a_{\sigma \tau}(\cdot) D^{\tau}\right) .
$$

Thus, partial integration on $C_{0}^{\infty}(\Omega) \times C_{0}^{\infty}(\Omega)$ gives the bilinear form $b(\cdot, \cdot)$ :

$$
b(u, \varphi):=\left(P\left(\cdot, D_{x}\right) u, \varphi\right)_{0 ; \Omega}=\sum_{\sigma, \tau \in \Gamma}\left(a_{\sigma \tau} D^{\tau} u, D^{\sigma} \varphi\right)_{0 ; \Omega} .
$$

Because of the boundedness of the functions $a_{\sigma \tau}$, there exists a constant $C \in \mathbf{R}^{+}$with

$$
|b(u, \varphi)| \leq C\|u\|_{1,1 ; \Omega}\|\varphi\|_{1,1 ; \Omega} \quad \text { for all } u, \varphi \in C_{0}^{\infty}(\Omega),
$$

and the bilinear form $b(\cdot, \cdot)$ can be continuously extended to $H_{0}^{1,1}(\Omega) \times H_{0}^{1,1}(\Omega)$. This extension will also be denoted by $b(\cdot, \cdot)$. Thus we can give the following weak formulation of Problem $(\Omega)$. 
Problem (B). For $f \in L^{2}(\Omega)$ find a function $u \in H_{0}^{1,1}(\Omega)$ such that

$$
b(u, \varphi)=(f, \varphi)_{0 ; \Omega} \quad \text { for all } \varphi \in H_{0}^{1,1}(\Omega) .
$$

We call the bilinear form $b(\cdot, \cdot) H_{0}^{1,1}(\Omega)$-coercive if there are constants $\varrho \in \mathbf{R}^{+}$ and $q \in \mathbf{R}$ with which

$$
b(u, u) \geq \varrho\|u\|_{1,1 ; \Omega}^{2}-q\|u\|_{0 ; \Omega}^{2} \quad \text { for all } u \in H_{0}^{1,1}(\Omega) .
$$

\section{Regularity results}

3.1. To show regularity for the solutions of the Problems $(\Omega)$ and (B) we shall use the classical elliptic theory. So let the domains $\Omega_{\mu}(\mu=1,2)$ and the uniformly elliptic operators $P_{\mu}\left(\cdot, D_{x_{\mu}}\right)$ be defined as in subsection 2.3. We then consider the following classical homogeneous elliptic Dirichlet problems:

Problem $\left(\Omega_{\mu}\right)(\mu=1,2)$. For $f_{\mu} \in C^{0 ; \lambda}\left(\Omega_{\mu}\right) \cap C\left(\bar{\Omega}_{\mu}\right)(\lambda \in(0,1])$ find a function $u_{\mu} \in C^{2}\left(\Omega_{\mu}\right) \cap C\left(\bar{\Omega}_{\mu}\right)$ such that

$$
\begin{gathered}
P_{\mu}\left(x_{\mu}, D_{x_{\mu}}\right) u_{\mu}\left(x_{\mu}\right)=f_{\mu}\left(x_{\mu}\right) \quad \text { for } x_{\mu} \in \Omega_{\mu}, \\
u_{\mu}\left(x_{\mu}\right)=0 \quad \text { for } x_{\mu} \in \partial \Omega_{\mu} .
\end{gathered}
$$

From C. Miranda [18], 21.VI, p. 80, and A. Friedman [7], Theorem 17.3, p. 68, one gets

Theorem 3.1. Let $\Omega_{\mu}$ be a bounded domain with boundary $\partial \Omega_{\mu}$ of class $C^{\infty}$. Let further Problem $\left(\Omega_{\mu}\right)$ be uniquely solvable. Then there exists a measurable Green's function $g_{\mu}: \Omega_{\mu} \times \Omega_{\mu} \rightarrow \overline{\mathbf{R}}(=\mathbf{R} \cup\{\infty\})$ such that the solution of Problem $\left(\Omega_{\mu}\right)$ is given by

$$
u_{\mu}\left(x_{\mu}\right):=\int_{\Omega_{\mu}} g_{\mu}\left(x_{\mu}, y_{\mu}\right) f_{\mu}\left(y_{\mu}\right) d y_{\mu} \quad \text { for } x_{\mu} \in \Omega_{\mu} .
$$

The function $g_{\mu}$ satisfies in the domain $\Omega_{\mu} \times \Omega_{\mu}$ the estimate

$$
\left|g_{\mu}\left(x_{\mu}, y_{\mu}\right)\right| \leq \text { const }\left|x_{\mu}-y_{\mu}\right|^{1-n_{\mu}} \text {. }
$$

For $f_{\mu} \in C^{\infty}\left(\bar{\Omega}_{\mu}\right)$ the solution $u_{\mu}$ of Problem $\left(\Omega_{\mu}\right)$ lies in the class $C^{\infty}\left(\bar{\Omega}_{\mu}\right)$. In this case there exists for each $k \in \mathbf{N}_{0}$ a constant $c_{k} \in \mathbf{R}^{+}$(independent of $f_{\mu}$ ) such that

$$
\left\|u_{\mu}\right\|_{k+2 ; \Omega_{\mu}} \leq c_{k}\left\|f_{\mu}\right\|_{k ; \Omega_{\mu}}
$$


3.2. The above theorem is now the main argument for the regularity of the solutions of Problem $(\Omega)$.

Theorem 3.2. Assume that Problems $\left(\Omega_{\mu}\right)(\mu=1,2)$ are uniquely solvable. Then there exists for each $f \in C^{\infty}(\bar{\Omega})$ a solution $u$ of Problem $(\Omega)$ with

(i) $u \in C^{\infty}(\bar{\Omega})$,

(ii) for each $(s, t) \in \mathbf{N}_{0}^{2}$ there exists a constant $c_{s t} \in \mathbf{R}^{+}$(independent of $\left.f \in C^{\infty}(\bar{\Omega})\right)$ such that

$$
\|u\|_{s+2, t+2 ; \Omega} \leq c_{s t}\|f\|_{s, t ; \Omega} .
$$

Furthermore, the boundary conditions (2.7) and (2.8) are satisfied for all multi-indices $\alpha^{\mu} \in \mathcal{N}^{\mu}$.

Proof. Take Green's function $g_{2}$ of Theorem 3.1,

$$
g_{2}: \Omega_{2} \times \Omega_{2} \rightarrow \overline{\mathbf{R}}, \quad\left(x_{2}, y_{2}\right) \mapsto g_{2}\left(x_{2}, y_{2}\right) .
$$

Fix $y_{1} \in \Omega_{1}$ and define a function $z\left(y_{1}, \cdot\right)$ by

$$
z\left(y_{1}, \cdot\right): \Omega_{2} \rightarrow \mathbf{R}, \quad x_{2} \mapsto \int_{\Omega_{2}} g_{2}\left(x_{2}, y_{2}\right) f\left(y_{1}, y_{2}\right) d y_{2} .
$$

Obviously $z\left(y_{1}, \cdot\right)$ is a solution of Problem $\left(\Omega_{2}\right)$. Since we have $f\left(y_{1}, \cdot\right) \in C^{\infty}\left(\bar{\Omega}_{2}\right)$ for all $y_{1} \in \Omega_{1}$ by assumption, we get $z\left(y_{1}, \cdot\right) \in C^{\infty}\left(\bar{\Omega}_{2}\right)$ by Theorem 3.1, and thus we have the function $z: \Omega_{1} \times \Omega_{2} \rightarrow \mathbf{R},\left(y_{1}, x_{2}\right) \mapsto z\left(y_{1}, x_{2}\right)$.

Now we show that

$$
D^{\alpha^{1}} z\left(y_{1}, \cdot\right) \in C^{\infty}\left(\bar{\Omega}_{2}\right) \quad \text { for all } y_{1} \in \Omega_{1} \text { and } \alpha^{1} \in \mathcal{N}^{1} .
$$

Fix $x_{2} \in \Omega_{2}$. Because of (3.2) and $f \in C^{\infty}(\bar{\Omega})$ we get for $\alpha^{1} \in \mathcal{N}^{1}$

$$
\left|g_{2}\left(x_{2}, y_{2}\right) D^{\alpha^{1}} f\left(y_{1}, y_{2}\right)\right| \leq \text { const }\left|x_{2}-y_{2}\right|^{1-n_{2}} \quad \text { for all } y_{2} \in \Omega_{2},
$$

which is valid uniformly on $\bar{\Omega}_{1}$. Since the right side of (3.7) is summable over $\Omega_{2}$, we get for all $y_{1} \in \Omega_{1}$

$$
D^{\varepsilon_{i}} \int_{\Omega_{2}} g_{2}\left(x_{2}, y_{2}\right) f\left(y_{1}, y_{2}\right) d y_{2}=\int_{\Omega_{2}} g_{2}\left(x_{2}, y_{2}\right) D^{\varepsilon_{i}} f\left(y_{1}, y_{2}\right) d y_{2}
$$

(cf. Dieudonné [3], 13.8 .6 , p. 124), whereby the partial derivatives $D^{\varepsilon_{i}}$ only act on the variable $y_{1}$. By induction with respect to the order of the multi-index $\alpha^{1}$ we further get

$$
D^{\alpha^{1}} z\left(y_{1}, x_{2}\right)=\int_{\Omega_{2}} g_{2}\left(x_{2}, y_{2}\right) D^{\alpha^{1}} f\left(y_{1}, y_{2}\right) d y_{2} \quad \text { for all } y_{1} \in \Omega_{1},
$$


and thus the partial derivatives $D^{\alpha^{2}} D^{\alpha^{1}} z$ of the function $z$ exist for all $\alpha^{\mu} \in \mathcal{N}^{\mu}$. From Theorem 3.1 and $f \in C^{\infty}(\bar{\Omega})$ we finally conclude $D^{\alpha^{1}} z\left(y_{1}, \cdot\right) \in C^{\infty}\left(\bar{\Omega}_{2}\right)$ for $y_{1} \in \Omega_{1}$ and $\alpha^{1} \in \mathcal{N}^{1}$.

Next we show that the functions $D^{\alpha^{2}} D^{\alpha^{1}} z$ are continuous and therefore measurable on $\Omega$. Fix $\left(y_{10}, x_{20}\right) \in \Omega$ and choose a sequence $\left\{\left(y_{1 m}, x_{2 m}\right)\right\}_{m \in N}$ of points in $\Omega$ converging to $\left(y_{10}, x_{20}\right) \in \Omega$. Then one gets by the triangle inequality

$$
\begin{aligned}
& \left|D^{\alpha^{2}} D^{\alpha^{1}} z\left(y_{10}, x_{20}\right)-D^{\alpha^{2}} D^{\alpha^{1}} z\left(y_{1 m}, x_{2 m}\right)\right| \\
& \leq\left|D^{\alpha^{2}} D^{\alpha^{1}} z\left(y_{10}, x_{20}\right)-D^{\alpha^{2}} D^{\alpha^{1}} z\left(y_{10}, x_{2 m}\right)\right| \\
& +\left|D^{\alpha^{2}} D^{\alpha^{1}} z\left(y_{10}, x_{2 m}\right)-D^{\alpha^{2}} D^{\alpha^{1}} z\left(y_{1 m}, x_{2 m}\right)\right|
\end{aligned}
$$

for $m \in \mathbf{N}$.

Since $D^{\alpha^{1}} z\left(y_{10}, \cdot\right) \in C^{\infty}\left(\bar{\Omega}_{2}\right)$, we have

$$
\left|D^{\alpha^{2}} D^{\alpha^{1}} z\left(y_{10}, x_{20}\right)-D^{\alpha^{2}} D^{\alpha^{1}} z\left(y_{10}, x_{2 m}\right)\right| \rightarrow 0
$$

for $m \rightarrow \infty$.

To estimate the second term in (3.9), we choose $t \in \mathbf{N}$ with $t>\frac{1}{2} n_{2}+\left|\alpha^{2}\right|$. By the Sobolev embedding theorem there is a constant $c \in \mathbf{R}^{+}$, independent of $m \in \mathbf{N}$, with

$$
\left\|D^{\alpha^{2}}\left(D^{\alpha^{1}} z\left(y_{10}, \cdot\right)-D^{\alpha^{1}} z\left(y_{1 m}, \cdot\right)\right)\right\|_{\infty ; \Omega_{2}} \leq c\left\|D^{\alpha^{1}} z\left(y_{10}, \cdot\right)-D^{\alpha^{1}} z\left(y_{1 m}, \cdot\right)\right\|_{t ; \Omega_{2}} .
$$

Now by definition we have

$$
D^{\alpha^{1}} z\left(y_{10}, x_{2}\right)-D^{\alpha^{1}} z\left(y_{1 m}, x_{2}\right)=\int_{\Omega_{2}} g_{2}\left(x_{2}, y_{2}\right) D^{\alpha^{1}}\left(f\left(y_{10}, y_{2}\right)-f\left(y_{1 m}, y_{2}\right)\right) d y_{2}
$$

for $x_{2} \in \Omega_{2}$. By Theorem 3.1 we get

$$
\left\|D^{\alpha^{1}} z\left(y_{10}, \cdot\right)-D^{\alpha^{1}} z\left(y_{1 m}, \cdot\right)\right\|_{t ; \Omega_{2}} \leq c_{t-2}\left\|D^{\alpha^{1}}\left(f\left(y_{10}, \cdot\right)-f\left(y_{1 m}, \cdot\right)\right)\right\|_{t-2 ; \Omega_{2}}
$$

and, since $f \in C^{\infty}(\bar{\Omega})$, it follows

$$
\left\|D^{\alpha^{1}}\left(f\left(y_{10}, \cdot\right)-f\left(y_{1 m}, \cdot\right)\right)\right\|_{t-2 ; \Omega_{2}} \rightarrow 0
$$

for $m \rightarrow \infty$ and thus

$$
\left|D^{\alpha^{2}} D^{\alpha^{1}} z\left(y_{10}, x_{2 m}\right)-D^{\alpha^{2}} D^{\alpha^{1}} z\left(y_{1 m}, x_{2 m}\right)\right| \rightarrow 0
$$


for $m \rightarrow \infty$. This shows the continuity of the functions $D^{\alpha^{2}} D^{\alpha^{1}} z$ in $\Omega$.

We next define for arbitrary chosen $t \in \mathbf{N}_{0}$ and $\alpha^{1} \in \mathcal{N}^{1}$ the function

$$
H: \Omega_{1} \rightarrow \mathbf{R}_{0}^{+}, \quad y_{1} \mapsto\left\|D^{\alpha^{1}} z\left(y_{1}, \cdot\right)\right\|_{t+2 ; \Omega_{2}}^{2}
$$

and show that

(i) $H$ is measurable on $\Omega_{1}$,

(ii) $H$ satisfies the estimate

$$
\int_{\Omega_{1}}\left|H\left(y_{1}\right)\right| d y_{1} \leq c_{t}^{2}\left\|D^{\alpha^{1}} f\right\|_{0, t ; \Omega}^{2} .
$$

To prove the first statement we note that the functions

$$
\left(y_{1}, x_{2}\right) \mapsto\left|D^{\alpha^{2}} D^{\alpha^{1}} z\left(y_{1}, x_{2}\right)\right|^{2}
$$

are measurable for all $\alpha^{\mu} \in \mathcal{N}^{\mu}$ on $\Omega$. From this we conclude (cf. Bauer [2], 22.6, p. 100) that the functions

$$
y_{1} \mapsto \int_{\Omega_{2}}\left|D^{\alpha^{2}} D^{\alpha^{1}} z\left(y_{1}, x_{2}\right)\right|^{2} d x_{2}
$$

are measurable on $\Omega_{1}$. Since each finite sum of measurable functions is measurable, we get (i).

To prove the second statement we replace (3.1) in Theorem 3.1 by (3.8) and get analogously to (3.3)

$$
\left\|D^{\alpha^{1}} z\left(y_{1}, \cdot\right)\right\|_{t+2 ; \Omega_{2}} \leq c_{t}\left\|D^{\alpha^{1}} f\left(y_{1}, \cdot\right)\right\|_{t ; \Omega_{2}} \quad \text { for all } y_{1} \in \Omega_{1}
$$

and thus

$$
\left|H\left(y_{1}\right)\right| \leq c_{t}^{2}\left\|D^{\alpha^{1}} f\left(y_{1}, \cdot\right)\right\|_{t ; \Omega_{2}}^{2} \quad \text { for all } y_{1} \in \Omega_{1} .
$$

The measurability of $H$ on $\Omega_{1}$ and the summability of $y_{1} \mapsto\left\|D^{\alpha^{1}} f\left(y_{1}, \cdot\right)\right\|_{t ; \Omega_{2}}^{2}$ over $\Omega_{1}$, together with the theorem of Fubini (cf. Bauer [2], Satz 22.6, p. 100), lead to

$$
\begin{aligned}
\left\|D^{\alpha^{1}} z\right\|_{0, t+2 ; \Omega} & =\left(\int_{\Omega_{1}}\left\|D^{\alpha^{1}} z\left(y_{1}, \cdot\right)\right\|_{t+2 ; \Omega_{2}}^{2} d y_{1}\right)^{1 / 2} \\
& =\left(\int_{\Omega_{1}}\left|H\left(y_{1}\right)\right| d y_{1}\right)^{1 / 2} \leq c_{t}\left\|D^{\alpha^{1}} f\right\|_{0, t ; \Omega} .
\end{aligned}
$$

Thus we get $D^{\alpha^{2}} D^{\alpha^{1}} z \in L^{2}(\Omega)$ for all $\alpha^{\mu} \in \mathcal{N}^{\mu}$ and therefore $z \in W^{k}(\Omega)$ for all $k \in \mathbf{N}_{0}$. By the Sobolev embedding we have $z \in C^{\infty}(\bar{\Omega})$. 
Finaily, we construct a solution of Problem $(\Omega)$ :

$$
u\left(x_{1}, x_{2}\right):=\int_{\Omega_{1}} g_{1}\left(x_{1}, y_{1}\right) z\left(y_{1}, x_{2}\right) d y_{1} \quad \text { for all }\left(x_{1}, x_{2}\right) \in \Omega_{1} \times \Omega_{2} .
$$

Obviously this function $u: \Omega \rightarrow \mathbf{R}$ is an element in $C^{\infty}(\bar{\Omega})$. For $\alpha^{2} \in \mathcal{N}^{2}$ we have analogously to (3.8)

$$
D^{\alpha^{2}} u\left(x_{1}, x_{2}\right)=\int_{\Omega_{1}} g_{1}\left(x_{1}, y_{1}\right) D^{\alpha^{2}} z\left(y_{1}, x_{2}\right) d y_{1} \quad \text { for all }\left(x_{1}, x_{2}\right) \in \Omega_{1} \times \Omega_{2}
$$

i.e., for each $x_{2} \in \Omega_{2}$ the function $D^{\alpha^{2}} u\left(\cdot, x_{2}\right)$ is the solution of Problem $\left(\Omega_{1}\right)$,

$$
\begin{aligned}
P_{1}\left(x_{1}, D_{x_{1}}\right) D^{\alpha^{2}} u\left(x_{1}, x_{2}\right) & =D^{\alpha^{2}} z\left(x_{1}, x_{2}\right) & & \text { for all } x_{1} \in \Omega_{1}, \\
D^{\alpha^{2}} u\left(x_{1}, x_{2}\right) & =0 & & \text { for all } x_{1} \in \partial \Omega_{1},
\end{aligned}
$$

from which (2.8) follows for all $\alpha^{2} \in \mathcal{N}^{2}$.

By definition (3.5) we have

$$
P_{2}\left(x_{2}, D_{x_{2}}\right) z\left(x_{1}, x_{2}\right)=f\left(x_{1}, x_{2}\right) \quad \text { for all }\left(x_{1}, x_{2}\right) \in \Omega_{1} \times \Omega_{2}
$$

and we further get

$$
P\left(x, D_{x}\right) u(x)=f(x) \quad \text { for all } x \in \Omega .
$$

The theorem of Fubini yields

$$
\begin{aligned}
u\left(x_{1}, x_{2}\right) & =\int_{\Omega_{1}} g_{1}\left(x_{1}, y_{1}\right) z\left(y_{1}, x_{2}\right) d y_{1} \\
& =\int_{\Omega_{1}} g_{1}\left(x_{1}, y_{1}\right)\left(\int_{\Omega_{2}} g_{2}\left(x_{2}, y_{2}\right) f\left(y_{1}, y_{2}\right) d y_{2}\right) d y_{1} \\
& =\iint_{\Omega_{1} \times \Omega_{2}} g_{1}\left(x_{1}, y_{1}\right) g_{2}\left(x_{2}, y_{2}\right) f\left(y_{1}, y_{2}\right) d y_{1} d y_{2}
\end{aligned}
$$

for each $\left(x_{1}, x_{2}\right) \in \Omega_{1} \times \Omega_{2}$, from which we get for $\alpha^{1} \in \mathcal{N}^{1}$ analogously to (3.15)

$$
D^{\alpha^{1}} u\left(x_{1}, x_{2}\right)=\int_{\Omega_{2}} g_{2}\left(x_{2}, y_{2}\right)\left(D^{\alpha^{1}} \int_{\Omega_{1}} g_{1}\left(x_{1}, y_{1}\right) f\left(y_{1}, y_{2}\right) d y_{1}\right) d y_{2},
$$

and from this follows

$$
D^{\alpha^{1}} u\left(x_{1}, x_{2}\right)=0 \quad \text { for all }\left(x_{1}, x_{2}\right) \in \Omega_{1} \times \partial \Omega_{2},
$$


i.e. (2.7), for all $\alpha^{1} \in \mathcal{N}^{1}$.

Thus we have proved that the function $u: \Omega \rightarrow \mathbf{R}$, defined by the product of the Green's functions $g_{\mu}$, $u\left(x_{1}, x_{2}\right)=\iint_{\Omega_{1} \times \Omega_{2}} g_{1}\left(x_{1}, y_{1}\right) g_{2}\left(x_{2}, y_{2}\right) f\left(y_{1}, y_{2}\right) d y_{1} d y_{2} \quad$ for $\left(x_{1}, x_{2}\right) \in \Omega_{1} \times \Omega_{2}$, is a solution of Problem $(\Omega)$.

The relations

$$
\left\|D^{\alpha^{2}} u\right\|_{s+2,0 ; \Omega} \leq c_{s}\left\|D^{\alpha^{2}} z\right\|_{s, 0 ; \Omega} \quad \text { for all } \alpha^{2} \in \mathcal{N}^{2}
$$

which are analogous to (3.13), yield by (3.13) the estimates (3.4) with $c_{s t}:=c_{s} c_{t}$ because of

$$
\begin{aligned}
\|u\|_{s+2, t+2 ; \Omega}^{2} & =\sum_{\left|\alpha^{2}\right| \leq t+2}\left\|D^{\alpha^{2}} u\right\|_{s+2,0 ; \Omega}^{2} \leq c_{s}^{2} \sum_{\left|\alpha^{2}\right| \leq t+2}\left\|D^{\alpha^{2}} z\right\|_{s, 0 ; \Omega}^{2} \\
& =c_{s}^{2} \sum_{\left|\alpha^{1}\right| \leq s}\left\|D^{\alpha^{1}} z\right\|_{0, t+2 ; \Omega}^{2} \leq c_{s}^{2} c_{t}^{2} \sum_{\left|\alpha^{1}\right| \leq s}\left\|D^{\alpha^{1}} f\right\|_{0, t ; \Omega}^{2} \\
& =c_{s}^{2} c_{t}^{2}\|f\|_{s, t ; \Omega}^{2} .
\end{aligned}
$$

We remark that the solution $u$ defined by the product of Green's functions is the only $C^{2,2 ; \lambda}(\Omega)$-solution $(\lambda \in(0,1])$ of Problem $(\Omega)$. Assuming that $u \in$ $C^{2,2 ; \lambda}(\Omega)$ is a non-trivial solution of Problem $(\Omega)$ for $f=0$, we define a function $v \in C^{2,0 ; \lambda}(\Omega)$ by the relation

$$
v\left(x_{1}, x_{2}\right):=P_{2}\left(x_{2}, D_{x_{2}}\right) u\left(x_{1}, x_{2}\right) \quad \text { for all }\left(x_{1}, x_{2}\right) \in \Omega_{1} \times \Omega_{2} .
$$

Because of the uniqueness of Problem $\left(\Omega_{2}\right)$ there is

$$
u\left(x_{1}, x_{2}\right)=\int_{\Omega_{2}} g_{2}\left(x_{2}, y_{2}\right) v\left(x_{1}, y_{2}\right) d y_{2} \quad \text { for all }\left(x_{1}, x_{2}\right) \in \Omega_{1} \times \Omega_{2}
$$

and therefore

$$
P_{1}\left(x_{1}, D_{x_{1}}\right) u\left(x_{1}, x_{2}\right)=\int_{\Omega_{2}} g_{2}\left(x_{2}, y_{2}\right) P_{1}\left(x_{1}, D_{x_{1}}\right) v\left(x_{1}, y_{2}\right) d y_{2}=0 .
$$

Now the uniqueness of Problem $\left(\Omega_{1}\right)$ gives for each $x_{2} \in \Omega_{2}$

$$
u\left(x_{1}, x_{2}\right)=0 \quad \text { for all } x_{1} \in \Omega_{1},
$$

and thus $u(x)=0$ for $x \in \Omega$. 
3.3. Now we are able to prove some regularity results for the weak Problem (B). Our proofs require that the domain $\Omega$ is star-shaped, i.e., there exist a point $x \in \Omega$ and a continuous function $h:\left\{y \in \mathbf{R}^{n}|| y \mid=1\right\} \rightarrow \mathbf{R}^{+}$with

$$
\Omega=\left\{y \in \mathbf{R}^{n} \backslash\{x\}|| y-x \mid<h\left(\frac{y-x}{|y-x|}\right)\right\} \cup\{x\} .
$$

It is easy to see that $\Omega$ is star-shaped if and only if $\Omega_{1}$ and $\Omega_{2}$ are star-shaped.

Theorem 3.3. For a star-shaped domain $\Omega$ every solution of the classical Problem $(\Omega)$ for a given $f \in C(\Omega) \cap L^{2}(\Omega)$ is also a solution of the weak Problem (B).

From this theorem it will be clear that the solution $u$ of Problem $(\Omega)$, constructed in the proof of Theorem 3.2, is a smooth solution of Problem (B) for a given $f \in C^{\infty}(\bar{\Omega})$.

Proof. Let $u \in C^{2,2}(\Omega) \cap C^{1}(\bar{\Omega})$ be a solution of Problem $(\Omega)$ belonging to a given function $f \in C(\Omega) \cap L^{2}(\Omega)$. Then for all $\varphi \in C_{0}^{\infty}(\Omega)$ one has

$$
b(u, \varphi)=\sum_{\sigma, \tau \in \Gamma}\left(a_{\sigma \tau} D^{\tau} u, D^{\sigma} \varphi\right)_{0 ; \Omega}=(P(\cdot, D) u, \varphi)_{0 ; \Omega}=(f, \varphi)_{0 ; \Omega} .
$$

Since $C_{0}^{\infty}(\Omega)$ is dense in $H_{0}^{1,1}(\Omega)$, we get

$$
b(u, \varphi)=(f, \varphi)_{0 ; \Omega}
$$

for all $\varphi \in H_{0}^{1,1}(\Omega)$. Thus we only need to show $u \in H_{0}^{1,1}(\Omega)$.

To do this, we extend the function $u$ by

$$
\tilde{u}(x)=\left\{\begin{array}{ll}
u(x) & \text { for } x \in \bar{\Omega} \\
0 & \text { for } x \in \mathbf{R}^{n} \backslash \bar{\Omega}
\end{array} .\right.
$$

Partial integration gives $\tilde{u} \in W^{1,1}\left(\mathbf{R}^{n}\right)$. As the domain $\Omega$ is star-shaped, the assertion $u \in H_{0}^{1,1}(\Omega)$ follows in the same way as in J. Rákosník [20], Lemma 6, p. 136.

If Problem (B) is uniquely solvable, it follows as a conclusion of Theorem 3.3 that there exists at most one solution of Problem $(\Omega)$.

To the end of this section we assume that the assumptions of Theorem 3.2 are fulfilled.

Corollary 3.4. If $\Omega$ is a star-shaped domain and if $f \in C^{\infty}(\bar{\Omega})$, there exists a solution $u$ of Problem (B) with

$$
u \in C^{\infty}(\bar{\Omega}) \cap H_{0}^{1,1}(\Omega)
$$

In the next theorem we formulate a weak regularity result for the weak Problem (B). 
Theorem 3.5. Suppose that the domain $\Omega$ is star-shaped, the bilinear form $b(\cdot, \cdot)$ is $H_{0}^{1,1}(\Omega)$-coercive and that Problem (B) is uniquely solvable. Then the solution $u$ belonging to a function $f \in H^{s, t}(\Omega)\left(s, t \in \mathbf{N}_{0}\right)$ satisfies

$$
u \in H^{s+2, t+2}(\Omega) \cap H_{0}^{1,1}(\Omega),
$$

and there is a constant $c_{s t} \in \mathbf{R}^{+}$, independent of $f \in H^{s, t}(\Omega)$, such that

$$
\|u\|_{s+2, t+2 ; \Omega} \leq c_{s t}\|f\|_{s, t ; \Omega} .
$$

Proof. For a function $f \in H^{s, t}(\Omega)$, there exists a sequence $\left\{f_{m}\right\}_{m \in N} \subset$ $C^{\infty}(\bar{\Omega})$ with

$$
\lim _{m \rightarrow \infty}\left\|f_{m}-f\right\|_{s, t ; \Omega}=0
$$

(cf. J. Rákosník [19], Theorem 2, p. 60).

For each $m \in \mathbf{N}$, Corollary 3.4 gives a (unique) solution

$$
u_{m} \in C^{\infty}(\bar{\Omega}) \cap H_{0}^{1,1}(\Omega)
$$

of Problem (B) belonging to $f_{m}$, i.e.

$$
b\left(u_{m}, \varphi\right)=\left(f_{m}, \varphi\right)_{0 ; \Omega} \quad \text { for all } \varphi \in H_{0}^{1,1}(\Omega) .
$$

Here the functions $u_{m}$ are the solutions of Problem $(\Omega)$ for $f_{m}$ as constructed in the proof of Theorem 3.2. Thus there is a constant $c_{s t} \in \mathbf{R}^{+}$, independent of $m \in \mathbf{N}$, such that

$$
\left\|u_{m}\right\|_{s+2, t+2 ; \Omega} \leq c_{s t}\left\|f_{m}\right\|_{s, t ; \Omega} .
$$

Analogously we have

$$
\left\|u_{m_{1}}-u_{m_{2}}\right\|_{s+2, t+2 ; \Omega} \leq c_{s t}\left\|f_{m_{1}}-f_{m_{2}}\right\|_{s, t ; \Omega} .
$$

Therefore $\left(u_{m}\right)_{m \in N}$ is a Cauchy sequence in $H^{s, t}(\Omega)$ and there exists a function $\tilde{u} \in H^{s+2, t+2}(\Omega)$ with

$$
\lim _{m \rightarrow \infty}\left\|\tilde{u}-u_{m}\right\|_{s+2, t+2 ; \Omega}=0 .
$$

Let $u \in H_{0}^{1,1}(\Omega)$ be the solution of Problem (B) belonging to the given function $f$. Then $u-u_{m}$ are the solutions of Problem (B) belonging to $f-f_{m}$, and because of the coerciveness and uniqueness one can show that there is a constant $c \in \mathbf{R}^{+}$ with

i.e.

$$
\left\|u_{m}-u\right\|_{0 ; \Omega} \leq c\left\|f_{m}-f\right\|_{0 ; \Omega},
$$

From the inequality

$$
\lim _{m \rightarrow \infty}\left\|u_{m}-u\right\|_{0 ; \Omega}=0
$$

$$
\|\tilde{u}-u\|_{0 ; \Omega} \leq\left\|\tilde{u}-u_{m}\right\|_{0 ; \Omega}+\left\|u_{m}-u\right\|_{0 ; \Omega}
$$

for all $m \in \mathbf{N}$ and from the estimates (3.20) und (3.21) it follows $u=\tilde{u}$ and therefore $u \in H^{s+2, t+2}(\Omega)$.

The assertion (3.18) is then an immediate conclusion of (3.19).

From Theorem 3.5 a further regularity result immediately follows: 
Theorem 3.6. If the assumptions of Theorem 3.5 are valid with $k-\left[\frac{1}{2} n\right]+1$ $\geq 0$ and if $f \in H^{k, k}(\Omega)$, the solution $u$ of Problem (B) satisfies

$$
u \in C^{k-\left[\frac{1}{2} n\right]+1}(\bar{\Omega}) \cap H_{0}^{1,1}(\Omega) .
$$

Proof. By Theorem 3.5 we have $u \in H^{k+2, k+2}(\Omega) \cap H_{0}^{1,1}(\Omega)$. By $H^{k+2, k+2}(\Omega)$ $\subset H^{k+2}(\Omega)$ and by the Sobolev embedding it follows $u \in C^{k-\left[\frac{1}{2} n\right]+1}(\bar{\Omega}) \cap H_{0}^{1,1}(\Omega)$.

We remark that, by some additional and quite restrictive geometrical conditions on the domains $\Omega_{\mu}$, one can directly get further regularity results by using J. Rákosník [20], Theorem 1, p. 129, and Adams [1], Theorem 5.4, pp. 97-98.

The next Theorem 3.7 tells that, under the assumptions of Theorem 3.6 with $k-\left[\frac{1}{2} n\right]+1 \geq 2$, solutions of Problem (B) are also solutions of Problem $(\Omega)$.

Theorem 3.7. For $u \in H_{0}^{1,1}(\Omega) \cap C^{1}(\bar{\Omega})$ one has

$$
\begin{array}{ll}
D^{\alpha^{1}} u(x)=0 & \text { for all } x \in \Omega_{1} \times \partial \Omega_{2}, \\
D^{\alpha^{2}} u(x)=0 & \text { for all } x \in \partial \Omega_{1} \times \Omega_{2}
\end{array}
$$

for $\alpha^{\mu} \in \mathcal{N}^{\mu}$ with $\left|\alpha^{1}\right|,\left|\alpha^{2}\right| \leq 1$ (cf. K. Doppel, N. Jacob [4], Theorem 4, p. 380).

\section{References}

[1] Adams, R.A.: Sobolev spaces. - Academic Press, New York-San Francisco-London, 1975.

[2] BaUer, H.: Wahrscheinlichkeitstheorie und Grundzüge der Maßtheorie. - Walter de Gruyter \& Co., Berlin, 1968.

[3] Dieudonné, J.: Treatise on analysis. II. - Academic Press, New York-London, 1970.

[4] Doppel, K., and N. JACOB: A non-hypoelliptic Dirichlet problem from stochastics. Ann. Acad. Sci. Fenn. Ser. A I Math. 8, 1983, 375-390.

[5] DopPeL, K., and N. JACOB: On an evolution equation for a non-hypoelliptic linear partial differential operator from stochastics. - Z. Anal. Anwendungen 3, 1984, 425-433.

[6] Dynkin, E.B.: Harmonic functions associated with several Markov processes. - Adv. in Appl. Math. 2, 1981, 260-283.

[7] Friedman, A.: Partial differential equations. - Holt, Rinehart and Winston, Inc., New York-Chicago-etc., 1969.

[8] Fujiwara, D., and H. OMORI: An example of a globally hypo-elliptic operator. - Hokkaido Math. J. 12, 1983, 293-297.

[9] Greenfield, S.J., and N.R. WALLACH: Global hypoellipticity and Liouville numbers. Proc. Amer. Math. Soc. 31, 1972, 112-114.

[10] HERrLer, H.-J.: Entartete lineare elliptische Differentialgleichungen und anisotrope Sobolevräume: Existenz schwacher Lösungen. - Z. Anal. Anwendungen 5, 1986, 223-236.

[11] HERRLER, H.-J.: Entartete lineare elliptische Differentialgleichungen und anisotrope Sobolevräume: Regularität schwacher Lösungen. - Z. Anal. Anwendungen 5, 1986, 533542.

[12] Hоснмuтн, R.: Regularitätsresultate für ein Randwertproblem einer nicht-hypoelliptischen linearen partiellen Differentialgleichung. - Dissertation, Freie Universität Berlin, 1989. 
[13] HöRMANDER, L.: The analysis of linear partial differential operators. II. - Springer-Verlag, Berlin-Heidelberg-etc., 1983.

[14] JАСов, N.: On generalized Dirichlet problems. - Math. Scand. 55, 1984, 245-252.

[15] LouhivaARA, I.S., and C.G. Simader: Über nichtelliptische lineare Differentialgleichungen mit konstanten Koeffizienten. - Ann. Acad. Sci. Fenn. Ser. A I Math. 513, 1972, 1-22.

[16] LouhivaARA, I.S., and C.G. Simader: Über periodische Lösungen koerzitiver linearer partieller Differentialgleichungen. - Ann. Acad. Sci. Fenn. Ser. A I Math. 4, 1978/79, 87-108.

[17] MANGERoN, D.: Sopra un problema al contorno per un'equazione differenziale alle derivate parziali di quart'ordine con le caratteristiche reali doppie. - Giornale di Matematiche di Battaglini 71 [(3) 24], Napoli, 1933, 89-139.

[18] Miranda, C.: Partial differential equations of elliptic type. - Springer-Verlag, BerlinHeidelberg-New York, 1970.

[19] RÁkosník, J.: Some remarks to anisotropic Sobolev spaces I. - Beiträge Anal. 13, 1979, 55-68.

[20] RÁkosNík, J.: Some remarks to anisotropic Sobolev spaces II. - Beiträge Anal. 15, 1981, 127-140.

[21] Schomburg, B.: Das verallgemeinerte homogene Dirichletproblem für Produkte linearer elliptischer Differentialoperatoren zweiter Ordnung. - Dissertation, Freie Universität Berlin, 1985.

[22] Wloka, J.: Partielle Differentialgleichungen. - B.G. Teubner, Stuttgart, 1982.

[23] Yoshino, M.: A class of globally hypoelliptic operators on the torus. - Math. Z. 201, 1989, 1-11.

Freie Universität Berlin

Institut für Mathematik I

Arnimallee 2-6

DW-1000 Berlin 33

Bundesrepublik Deutschland

Received 6 August 1990 